RAIRO Operations Research

RAIRO Oper. Res. 40 (2006) 37-52

DOI: $10.1051 /$ ro:2006008

\title{
AN ANALYTIC CENTER CUTTING PLANE ALGORITHM FOR FINDING EQUILIBRIUM POINTS*
}

\author{
FERnANDA M.P. RaupP ${ }^{1}$ And Wilfredo SosA ${ }^{2}$
}

\begin{abstract}
We present a variant of the analytic center cutting plane algorithm proposed by Goffin et al. (1996) to approximately solve equilibrium problems as proposed by Blum and Oettli (1994), which include as particular problems the variational inequalities problem, the Nash equilibria problem in non-cooperative games, the convex minimization problem, and the fixed point problem. Furthermore, we analyze the convergence and complexity of the modified algorithm.
\end{abstract}

Keywords. Equilibrium problems, convex feasibility problem, analytic center cutting plane algorithm.

Mathematics Subject Classification. 90C25, 90C51.

\section{INTRODUCTION}

In this work we deal with the equilibrium problem in the following format:

$$
\text { (EP) find } \bar{x} \in K \text { such that } f(\bar{x}, y) \geq 0 \forall y \in K \text {, }
$$

where $f:[0,1]^{n} \times[0,1]^{n} \rightarrow \mathbb{R}$ satisfies the following properties:

P1. $f(x, x)=0 \forall x \in[0,1]^{n}$;

P2. $f(x, \cdot):[0,1]^{n} \rightarrow \mathbb{R}$ is convex and lower semi-continuous $\forall x \in[0,1]^{n}$;

P3. $f(\cdot, y):[0,1]^{n} \rightarrow \mathbb{R}$ is upper semicontinuous $\forall y \in[0,1]^{n}$;

and $K$ is a nonempty compact convex subset of $[0,1]^{n}$.

Received July 10, 2004. Accepted November 8, 2005.

* The authors are thankful for the support of Finep/PRONEX-LNCC N. 76.97.0999.00.

${ }^{1}$ Laboratório Nacional de Computação Científica - MCT, Av. Getúlio Vargas, 333, Petrópolis, RJ, CEP 25651-075, Brazil; fernanda@lncc.br

2 Instituto de Matemática e Ciencias Afines - Universidad Nacional de Ingeniería, Jirón Ancash 536, Lima 1, Lima, Peru; sosa@uni.edu.pe

(c) EDP Sciences 2006 
As the set $K$ is bounded, we can consider the hypercube $[0,1]^{n}$ as its containing set without loss of generality, since we can always scale the variables.

The EP general formulation (1) with the name "equilibrium problem" was introduced first by Blum and Oettli [4]. However, the same formulation with another name was addressed before by Nikaido and Isoda [15], and then by Fan [7]. It is also important to cite the work of Brezis et al. [6], which extends the results of [7] for $K$ not compact.

It has been pointed out in $[4,12,13]$ that EP general formulation includes as particular problems the convex minimization problem, the fixed point problem, the Nash equilibria problem for noncooperative games and the variational inequalities problem. Moreover, EP is closest in formulation to the equilibrium programming problem introduced by Antipin and Vasil'ev [1]. Hereafter, a solution of EP (1) is called an equilibrium point.

As a matter of completion, next we show how the four problems mentioned above can be formulated as particular cases of EP. Assuming $K$ is a nonempty closed convex subset of $\mathbb{R}^{n}$, we exhibit appropriate function $f$ for each particular problem such that its solution set coincides with the solution set of EP. One can show that properties P1, P2 and P3 hold.

a) The convex minimization problem. Let $h: K \rightarrow \mathbb{R}$ be a convex and lower semicontinuous function. The convex minimization problem which is formulated as follows:

$$
\text { find } x \in K \text { such that } h(x) \leq h(y) \forall y \in K
$$

can be reformulated as an equilibrium problem taking $f(x, y):=h(y)-h(x)$ for all $x, y \in K$.

b) The fixed point problem. Let $T: \mathbb{R}^{n} \rightarrow \mathcal{P}\left(\mathbb{R}^{n}\right)$ be an upper semicontinuous point-to-set mapping such that $T(x) \cap K$ is a nonempty compact convex set for each $x \in K$. The fixed point problem is defined as:

$$
\text { find } x \in K \text { such that } x \in T(x) \text {. }
$$

Our goal is accomplished by setting $f(x, y):=\max _{u \in T(x) \cap K}(x-u)^{T}(y-x)$ for all $x, y \in K$.

c) The Nash equilibria problem in noncooperative games. Let $I$ be a finite set (the set of players). For each $i \in I$, consider a nonempty compact convex subset $K_{i}$ of $\mathbb{R}^{n}$ (the strategy set of the $i$ th player). Let $K:=\prod_{i \in I} K_{i}$. For each $i \in I$ consider a continuous function $f_{i}: K \rightarrow \mathbb{R}$ (the loss function of the $i$ th player, depending on the strategies of all players) which is convex in the $i$ th variable. For each $x, y \in K$, we define $x\left(y_{i}\right)$ as $\left(x\left(y_{i}\right)\right)_{j}:=x_{j}$ for all $j \neq i$, and $\left(x\left(y_{i}\right)\right)_{i}:=y_{i}$. The Nash equilibria problem in noncooperative games is formulated as:

find $x \in K$ such that $f_{i}(x) \leq f_{i}\left(x\left(y_{i}\right)\right) \quad \forall i \in I \quad \forall y \in K$. 
In this case, set $f(x, y):=\sum_{i \in I}\left(f_{i}\left(x\left(y_{i}\right)\right)-f_{i}(x)\right)$ for all $x, y \in K$ to obtain the EP general formulation.

d) The variational inequalities problem. Let $T: K \rightarrow \mathcal{P}\left(\mathbb{R}^{n}\right)$ be an upper semicontinuous point-to-set mapping such that $T(x)$ is a compact set for all $x \in K$. The variational inequality problem is defined as:

find $x \in K, u \in T(x)$, such that $u^{T}(y-x) \geq 0 \forall y \in K$.

Now, take $f(x, y):=\max _{u \in T(x)} u^{T}(y-x)$ for all $x, y \in K$.

Considering the general formulation EP, existing algorithms for finding approximately equilibrium points exploit orthogonal projection techniques and use hypotheses such as continuity, differentiability and/or monotonicity on $f$, as we can observe as follows.

Resembling the extragradient method of Korpelevich [11], Antipin [2] in his prediction gradient method generates two sequences:

$$
\begin{aligned}
& y^{k}=P_{K}\left(x^{k}-\alpha \nabla_{2} f\left(x^{k}, x^{k}\right)\right),{ }^{1} \\
& x^{k+1}=P_{K}\left(x^{k}-\alpha \nabla_{2} f\left(y^{k}, y^{k}\right)\right) .
\end{aligned}
$$

The hypotheses considered on $f$ such as differentiability in $K$ and monotonicity (i.e. $f(x, y)+f(y, x) \leq 0$ for all $x, y \in K)$, enables Antipin to show that the generated sequence $\left\{x^{k}\right\}$ converges to a point in the solution set of EP.

Recall that $K$ is a nonempty compact convex subset of $[0,1]^{n}$. For $y \in K$, let us define the following set

$$
L_{f}(y)=\left\{x \in[0,1]^{n}: f(y, x) \leq 0\right\} .
$$

Assuming continuity and pseudomonotonicity of $f$, the algorithm proposed by Iusem and Sosa [13] generates also two sequences as follows:

$$
\begin{aligned}
& y^{k} \text { such that } \max _{y \in K_{k}} f\left(y, x^{k}\right) \leq f\left(y^{k}, x^{k}\right)+\epsilon_{k}, \\
& x^{k+1}=x^{k}+\lambda_{k}\left(P_{L_{f}\left(y^{k}\right)}\left(x^{k}\right)-x^{k}\right),
\end{aligned}
$$

where

$$
K_{k}:=\left\{x \in K:\|x\| \leq \max \left\{\left\|x^{0}\right\|, \ldots,\left\|x^{k-1}\right\|\right\}+1\right\},
$$

and $\epsilon_{k}>0$ with $\lim _{k \rightarrow \infty} \epsilon_{k}=0$. Considering that $\left\{x^{k}\right\}$ or $\left\{y^{k}\right\}$ is bounded, they proved that the sequence $\left\{x^{k}\right\}$ generated by the algorithm converges to an equilibrium point.

In this paper we present a different approach to find equilibrium points approximately. Note that $L_{f}(y)$ is convex by property P2. Now, consider the definition of the following set

$$
S=\left(\cap_{y \in K} L_{f}(y)\right) \cap K .
$$

\footnotetext{
${ }^{1} P_{K}$ denotes the orthogonal projection over the set $K$ and $\nabla_{2} f$ denotes the gradient of $f$ with respect to the second argument.
} 
The set $S$ is contained in the solution set of EP, as we can see in [12] for example. So, the problem of finding a point in $S$ is a particular case of the convex feasibility problem, which in general consists of finding a point belonging to the intersection of a family of convex sets, for more information see [5]. The approach considered in this work is devised for finding a solution of EP by finding a point in $S$. No continuity, no differentiability and no monotonicity assumptions on $f$ are made. Nor any projection technique is used.

Here we study the application of a variant of the analytic center cutting plane algorithm for solving convex feasibility problems of Goffin et al. [8]. The first optimization method that combines the cutting plane methods with the technique of interior point methods was introduced by Sonnevend [17]. Besides allowing for feasible computer implementation, the methods developed with this characteristic have shown great performance in practice, see Goffin et al. [9], and Raupp and Gonzaga [16] for example.

The proposed method consists of building an increasingly refined polyhedral approximation of $S$. The linear inequalities that define the approximation of $S$ are generated by an oracle called at a query point as hyperplanes. In this version the query point is an approximation of the analytic center for the current polyhedral relaxation and the hyperplanes are originated from shallow cuts, which in turn makes the test point always an interior point for the new refined polyhedral approximation. Although its possible to add more than one cut per iteration as in Ye [20], the proposed method adds just one cut per iteration.

We also verify that the modified analytic center cutting plane method when applied to find an equilibrium point approximately is polynomial, with the complexity bound depending on the space dimension as in [8].

It is well known that a point close to the solution set of EP (an approximate solution of EP) can generate instability for methods using perturbations of the objective function and of the feasible set, see for example [1]. This is not the case of our approach. Since $S$ is compact and convex, we use the proposed analytic center cutting plane method in order to approximate $S$ by compact polyhedral sets, which contain the set $S$ and have nonempty interior. With this technique, we obtain in some finite iteration an approximate solution, since the volumes of the polyhedral sets tend to zero monotonically $[8,19]$.

The paper is organized as follows. We conclude the Introduction presenting the notation used hereafter. Section 2 briefly introduces some definitions and results from the theory of interior point methods. Section 3 is devoted to state some definitions and results related to the equilibrium problem already introduced. Under certain assumptions the proposed algorithm as well as its convergence analysis are presented in Section 4. Finally, in Section 5 we conclude our work with final comments.

\subsection{Notations}

Let $w$ and $z$ be $m$-dimensional real vectors. The matrices $W$ and $Z$ denote diagonal matrices with diagonals $w$ and $z$, respectively. Consequently, $W z$ denotes 
the component-wise product of $w$ and $z$. The vector $e$ denotes the vector with entries equal to 1 and dimension given by the context.

Given a nonempty closed set $K, \partial K$ denotes the boundary of $K$ and $\stackrel{o}{K}$ denotes its interior set. For each $x \in \mathbb{R}^{n}, P_{K}(x)$ denotes the orthogonal projection of the point $x$ over the set $K$ as already mentioned.

\section{AnAlytic CEnTER}

In this section we briefly recall some definitions and results from the theory of interior point methods. A plain exposition of such theory can be found in Ye [21], for example. We begin with the definition of the analytic center of a bounded polyhedral set and end with the presentation of the nature of the cutting planes used in the proposed technique for finding an approximate solution of EP.

Let $P \subseteq[0,1]^{n}$ be a bounded polyhedral set defined by $m(>n)$ linear inequalities given by

$$
P=\left\{x \in \mathbb{R}^{n}: A^{T} x \leq b\right\},
$$

where $A \in \mathbb{R}^{n \times m}$ with full row rank and $b \in \mathbb{R}^{m}$ are known, such that $P$ is nonempty and its set of interior points is given by

$$
\stackrel{o}{P}=\left\{x \in \mathbb{R}^{n}: A^{T} x<b\right\} .
$$

We assume that $P$ has nonempty interior in order to define the dual potential function $\phi: \stackrel{o}{P} \rightarrow \mathbb{R}$, given by

$$
\phi(x)=\sum_{j=1}^{m} \ln \left(b_{j}-a_{j}^{T} x\right),
$$

where $b_{j}$ is the $j$ th component of vector $b$ and $a_{j}$ is the $j$ th column of matrix $A$, $j=1, \ldots, m$. Note that $\phi(\cdot)$ decreases indefinitely as $x$ approaches $\partial P$.

Now, defining the dual slack vector by

$$
z=b-A^{T} x,
$$

the gradient and the Hessian of $\phi(\cdot)$ at $x$ are given respectively by

$$
\nabla \phi(x)=-A Z^{-1} e \quad \text { and } \quad \nabla^{2} \phi(x)=-A Z^{-2} A^{T},
$$

where $A Z^{-2} A^{T}$ is symmetric and positive definite.

Since $P$ is compact, $\stackrel{o}{P}$ is not empty and $\phi(\cdot)$ is strictly concave in $\stackrel{o}{P}$, the analytic center of $P$ defined by

$$
x^{a}=\operatorname{argmax}\{\phi(x): x \in \stackrel{o}{P}\}
$$


satisfies the first order optimality condition $\nabla \phi\left(x^{a}\right)=0$, i.e., there exists a Lagrangean multiplier $w^{a}>0$ such that

$$
A w^{a}=0, \quad z^{a}=b-A^{T} x^{a}>0 \quad \text { and } \quad W^{a} z^{a}=e .
$$

Given an interior point $x$ of $P$, we measure the proximity of this point to the analytic center $x^{a}$ using dual approach (evaluating the norm of a scaled gradient vector) which is

$$
\delta(z)=\left\|Z^{-1} A^{T}\left(A Z^{-2} A^{T}\right)^{-1} A Z^{-1} e\right\|=\|Z w(z)-e\|,
$$

where

$$
w(z):=Z^{-1}\left[I-Z^{-1} A^{T}\left(A Z^{-2} A^{T}\right)^{-1} A Z^{-1}\right] e,
$$

satisfies $A w(z)=0$ and $w(z)>0$.

Let $(x, z, w)$ be such that $z=b-A^{T} x$ and $w=w(z)$. The vector $x$ is an $\eta$-approximate analytic center of $P$ if

$$
\begin{aligned}
\|Z w-e\| & \leq \eta<1 \\
A^{T} x+z & =b, z>0 \\
A w & =0, w>0 .
\end{aligned}
$$

Given $x \in \stackrel{o}{P}$, the dual Newton method generates a new iterate

$$
x^{+}=x+\mathrm{d} x,
$$

where

$$
\mathrm{d} x=-\left[\nabla^{2} \phi(x)\right]^{-1} \nabla \phi(x)=-\left(A Z^{-2} A^{T}\right)^{-1} A Z^{-1} e,
$$

which was first proposed by Vaidya in [18]. Setting $z^{+}=b-A^{T} x^{+}$and $w^{+}=w\left(z^{+}\right)$, if $x$ is an $\eta$-approximate analytic center of $P$, then by Lemma 5.4 in Gonzaga [10] we get $z^{+}>0$ and $\left\|Z^{+} w^{+}-e\right\| \leq \eta^{2}$.

If $\left(x^{a}, z^{a}, w^{a}\right)$ satisfies (4) and $\left(x^{+}, z^{+}, w^{+}\right)$satisfies (5), then by Lemma 2 in Ye [19] we have that

$$
\phi\left(x^{a}\right) \geq \phi\left(x^{+}\right) \geq \phi\left(x^{a}\right)-\frac{\eta^{2}}{2(1-\eta)} .
$$

Let $\left(x^{+}, z^{+}, w^{+}\right)$be such that $x^{+}$is an $\eta$-approximate analytic center, $z^{+}=b-A^{T} x^{+}$and $w^{+}=w\left(z^{+}\right)$. Consider the following ellipsoid inscribing in $P$ with center at $x^{+}$

$$
E^{+}=\left\{x \in \mathbb{R}^{n}:\left\|\left(Z^{+}\right)^{-1} A^{T}\left(x-x^{+}\right)\right\| \leq 1-\eta\right\},
$$

denoted by Dikin ellipsoid.

Now, assume that $S(3)$ is defined implicitly by an oracle. The oracle either returns $x^{+} \in S$ or generates a hyperplane, which intersects the interior of the Dikin ellipsoid, as we can verify by the following lemma. 
Lemma 2.1. If $a \in \mathbb{R}^{n}$ with $\|a\|=1, \quad \tau:=\sqrt{a^{T}\left(A\left(Z^{+}\right)^{-2} A^{T}\right)^{-1} a}$, $\rho:=\sqrt{a^{T} A\left(Z^{+}\right)^{-2} A^{T} a}$ and $\left.\beta \in\right] 0, \frac{1-\eta}{\rho \tau}[$, then

$$
H^{+}=\left\{x \in \mathbb{R}^{n}: a^{T}\left(x-x^{+}\right)=\beta \tau\right\}
$$

intersects the interior of the Dikin ellipsoid.

Proof. Let $x^{p}$ be the projection of $x^{+}$on $H^{+}$, then $x^{p}=x^{+}+\beta \tau a$. So, we have $\left\|\left(Z^{+}\right)^{-1} A^{T}\left(x^{p}-x^{+}\right)\right\|=\left\|\left(Z^{+}\right)^{-1} A^{T}(\beta \tau a)\right\|=\beta \rho \tau<1-\eta$.

When the hyperplane $H^{+}(8)$ is added to the system, the new convex set is defined by

$$
\bar{P}=\left\{x \in \mathbb{R}^{n}: A^{T} x \leq b, a^{T} x \leq a^{T} x^{+}+\beta \tau\right\} .
$$

Now, denoting by $\bar{x}^{a}$ the analytic center of $\bar{P}$ and using Theorem 2 of Ye [19], one can show that the potential function $\phi(\cdot)$ satisfies

$$
\phi\left(\bar{x}^{a}\right) \leq \phi\left(x^{a}\right)+\ln (4 \tau)-(1.5-\beta)
$$

with $\tau$ and $\beta$ as defined in Lemma 2.1.

It is interesting to observe that $x^{+}$is still in the "quadratic convergence" region of $\bar{P}$ (Lem. 3 in Ye [19]). As a result, Goffin et al. [8] showed that it is necessary no more than four iterations of the dual Newton method (6) to find a new $\eta$-approximate analytic center of $\bar{P}$ starting from $x^{+}+\Delta x$, where

$$
\Delta x:=-\frac{\alpha(1-\eta)}{\tau}\left(A\left(Z^{+}\right)^{-2} A^{T}\right)^{-1} a
$$

is the affine direction that maximizes the new slack constrained to the Dikin ellipsoid trust region, and appropriated given values for $\alpha, \eta \in] 0,1[$.

\section{EQUiLibRium PROBLEM}

In this section, we characterize a solution and an approximate solution of EP. Some of the theoretical results presented here will be used in the next section.

Recall the sets $L_{f}(x)=\left\{y \in[0,1]^{n}: f(x, y) \leq 0\right\}$ (2) defined for each $x \in[0,1]^{n}$, and $S=\left(\cap_{x \in K} L_{f}(x)\right) \cap K(3)$, and consider the following definitions:

D1. the map $\Gamma:[0,1]^{n} \rightrightarrows \mathbb{R}^{n}$ given by

$$
\Gamma(x)=\left\{v \in \mathbb{R}^{n}: f(x, y) \geq v^{T}(y-x) \forall y \in[0,1]^{n}\right\}
$$

D2. the selection $T:[0,1]^{n} \rightarrow \mathbb{R}^{n}$ of $\Gamma$, i.e.

$$
T(x) \in \Gamma(x) \quad \forall x \in[0,1]^{n} .
$$

Observe that $\Gamma(x) \neq \emptyset \forall x \in] 0,1\left[{ }^{n}\right.$. 
Lemma 3.1. Let $\left\{x^{k}\right\}_{k \in \mathbb{N}} \subset K$ and $\left\{u^{k}\right\}_{k \in \mathbb{N}} \subset \mathbb{R}^{n}$ be two sequences such that $x^{k} \rightarrow \bar{x}$ and $u^{k} \rightarrow \bar{u}$. If $u^{k} \in \Gamma\left(x^{k}\right) \forall k \in \mathbb{N}$, then $\bar{u} \in \Gamma(\bar{x})$.

Proof. Since $u^{k} \in \Gamma\left(x^{k}\right) \forall k \in \mathbb{N}$, then $\left\langle u^{k}, x-x^{k}\right\rangle \leq f\left(x^{k}, x\right) \forall x \in K, k \in \mathbb{N}$. It implies that $\langle\bar{u}, x-\bar{x}\rangle \leq f(\bar{x}, x) \forall x \in K$, and so $\bar{u} \in \Gamma(\bar{x})$.

Theorem 3.2. If for each $x \in K, f(x, \cdot)$ is subdifferentiable at $x$, then the following statements hold:

(1) If $\bar{x} \in S$ then $\bar{x} \in K$ and $T(y)^{T}(\bar{x}-y) \leq 0 \forall y \in K$.

(2) If $\bar{x} \in K$ and $T(y)^{T}(\bar{x}-y) \leq 0 \forall y \in K$, then $\bar{x}$ is a solution of EP.

Proof.

1. If $\bar{x} \in S$, then $f(y, \bar{x}) \leq 0 \forall y \in K$. Since $T(y) \in \Gamma(y) \forall y \in K$, we have $T(y)^{T}(\bar{x}-y) \leq f(y, \bar{x}) \leq 0 \forall y \in K$.

2. If $\bar{x} \in K$ and $\bar{T}(y)^{T}(\bar{x}-y) \leq 0 \forall y \in K$. Take $y \in K$ arbitrarily and for each $\lambda \in] 0,1[$ consider $u:=\bar{\lambda} y+(1-\lambda) \bar{x}$, then $u \in K, \forall \lambda \in] 0,1[$. Thus, we have $0=T(u)^{T}(u-u)=\lambda T(u)^{T}(y-u)+(1-\lambda) T(u)^{T}(\bar{x}-u)$. By hypothesis $\left.T(u)^{T}(\bar{x}-u) \leq 0 \forall \lambda \in\right] 0,1\left[\right.$, which implies that $0 \leq T(u)^{T}(y-$ $u) \leq f(u, y) \forall \lambda \in] 0,1[$, and the statement follows from property P3.

The next theorem replaces the hypothesis of the previous one in order to reach the same goal.

Theorem 3.3. If for each $x \in K$ and $y \in \partial K$ there exists $\left\{y^{i}\right\}_{i \in \mathbb{N}} \subset \stackrel{o}{K}$ such that $y^{i} \rightarrow y$ and $f(x, y)=\lim _{i \rightarrow+\infty} f\left(x, y^{i}\right)$, then the following statements hold:

(1) If $\bar{x} \in S$, then $\bar{x} \in K$ and $T(y)^{T}(\bar{x}-y) \leq 0 \forall y \in \stackrel{o}{K}$.

(2) If $\bar{x} \in K$ and $T(y)^{T}(\bar{x}-y) \leq 0 \forall y \in \stackrel{o}{K}$, then $\bar{x}$ is a solution of EP.

Proof. Item 1 follows from item 1 of Theorem 3.2. For item 2, take $y \in \stackrel{o}{K}$ arbitrarily, then $\forall \lambda \in] 0,1[$ we have that $u:=\lambda y+(1-\lambda) \bar{x} \in \stackrel{o}{K}$. So, using the same argument as in item 2 of Theorem 3.2, we have that $0 \leq f(\bar{x}, y)$. Now, if $y \in \partial K$ the statement follows from the hypothesis.

The following lemma gives sufficient conditions in order to guarantee the hypothesis used in Theorems 3.2 and 3.3.

Lemma 3.4. If $K \subset] 0,1\left[{ }^{n}\right.$ and $\stackrel{o}{K} \neq \emptyset$, then

a) for each $x \in K, f(x, \cdot)$ is subdifferentiable at $x$;

b) for each $y \in \partial K$, there exists $\left\{y^{i}\right\}_{i \in \mathbb{N}} \subset \stackrel{o}{K}$ such that $y^{i} \rightarrow y$, then

$$
\lim _{i \rightarrow \infty} f\left(x, y^{i}\right)=f(x, y)
$$

c) if $f$ is bounded above on $K \times K$, then for each compact set $C \subset K$, $\cup_{x \in C} \Gamma(x)$ is bounded. 
Proof. Items a) and b) follow from the fact that each convex function is continuous and subdifferentiable at each interior point of its effective domain. For item c), take $\epsilon>0$ such that $\left.C(\epsilon):=\cup_{x \in C} B(x, \epsilon) \subset\right] 0,1\left[{ }^{n}\right.$, where $B(x, \epsilon):=\left\{y \in \mathbb{R}^{n}\right.$ : $\|y-x\| \leq \epsilon\}$. Given $u \in \cup_{x \in C} \Gamma(x)$, then there exists $x \in C$ such that $u \in \Gamma(x)$. So, $\left.u^{T}(y-x) \leq f(x, y) \forall y \in\right] 0,1\left[{ }^{n}\right.$. If $\|u\|>\epsilon$, take $z:=P_{B(x, \epsilon)}(x+u)$, then $u^{T}(z-x)=\|u\| \epsilon$. It implies that $\|u\| \epsilon \leq f(x, z)$. Finally, the statement follows from the boundedness of $f$.

Now let us define the following set for $\delta>0$ :

$$
S(\delta)=\cup_{x \in S} B(x, \delta) .
$$

Theorem 3.5. Let $f$ be bounded above on $K \times K$ and let $T:] 0,1\left[{ }^{n} \rightarrow \mathbb{R}^{n}\right.$ be a selection of $\Gamma$. Given $\epsilon>0$, there exists $\delta>0$ such that $\forall x \in S(\delta)$, we have that $T(y)^{T}(x-y) \leq \epsilon \forall y \in K$.

Proof. Let $L>0$ be such that $L:=\sup \left\{\|v\|: v \in \cup_{x \in K} \Gamma(x)\right\}$ and $\delta:=\epsilon / L$. Given $x \in S(\delta)$, we have that $T(y)^{T}(x-y)=T(y)^{T}\left(x-P_{S}(x)\right)+T(y)^{T}\left(P_{S}(x)-y\right) \leq$ $T(y)^{T}\left(x-P_{S}(x)\right) \leq L \delta=\epsilon, \forall y \in K$.

Definition 3.6. We say $x \in[0,1]^{n}$ is an $\epsilon$-solution for EP if and only if exists $\delta>0$ such that $d(x, S):=\inf _{y \in S}\|x-y\| \leq \delta$ and $T(y)^{T}(x-y)<\epsilon \forall y \in K$.

\section{Algorithm}

In this section, we start giving the assumptions made to present the proposed algorithm, which is a variant of the analytic center cutting plane algorithm in [8]. Finally, we analyze its convergence following [8].

For the algorithm we need to consider the listed assumptions:

A1. $S$ is nonempty;

A2. $f$ is bounded above on $K \times K$ and satisfies P1, P2, P3;

A3. there exists an oracle such that for each $x \in] 0,1\left[^{n}\right.$ it checks the inclusion

$$
x \in \operatorname{argmin}\left\{f(x, y): y \in[0,1]^{n}\right\} .
$$

If the inclusion is false, it generates $T(x) \in \Gamma(x)$;

A4. given $\bar{x} \in K$ and $x^{*} \in S$, if $f\left(\bar{x}, x^{*}\right)=0$ then $\bar{x}$ is a solution of EP.

We should emphasize that assumptions A1, A2, A4, P1, P2 and P3 are satisfied by a large class of functions $f$, meaning that some of them are not necessarily continuous nor monotone. For example, considering $K=[0,1]^{2}$, the following function

$$
f(x, y)= \begin{cases}1-y / x, & x>0 \\ 0, & x=0\end{cases}
$$

satisfies the assumptions $\mathrm{A} 1, \mathrm{~A} 2, \mathrm{~A} 4, \mathrm{P} 1, \mathrm{P} 2$ and $\mathrm{P} 3$, however $f$ is not continuous neither monotone (not even pseudomonotone). 
The assumption A1 holds if $f$ is properly quasimonotone and satisfies P1, P2 and P3. (This concept was introduced by Zhou-Chen in 1998 for the case of variational inequalities and then extended to bifunctions, for more information see [3].) The assumption A4 holds if $f(\cdot, y)$ has a unique maximizer for each $y \in K$.

The following lemma tells us that the hypothesis A4 is obtained if we use other classical ones for the particular problems considered in Section 1.

Lemma 4.1. Consider the four particular problems of EP presented in Section 1.

a) Given the convex minimization problem, the assumption A4 holds.

b) Given the fixed point problem, if $(-T)$ is monotone (i.e., $(u-v)^{T}(x-$ $y) \leq 0, \forall x, y \in K, u \in T(x), v \in T(y))$, then A4 holds.

c) Given the Nash equilibria problem in noncooperative games, if for each $i \in I, f_{i}$ is additive (i.e., $f_{i}(x)=\sum_{j \in I} f_{i j}\left(x_{j}\right), \forall x \in K$ ), then A4 holds.

d) Given the variational inequality problem. If $(T-I)$ is monotone, then $A_{4}$ holds.

Proof. Given $\bar{x} \in K$ and $x^{*} \in S$ such that $f\left(\bar{x}, x^{*}\right)=0$, it follows:

a) $0=f\left(\bar{x}, x^{*}\right)=h\left(x^{*}\right)-h(\bar{x})$. Hence $h(\bar{x})=h\left(x^{*}\right) \leq h(x) \forall x \in K$, and so $\bar{x}$ is a solution of EP.

b) $0=f\left(\bar{x}, x^{*}\right)=\sup _{u \in T(\bar{x}) \cap K}(\bar{x}-u)^{T}\left(x^{*}-\bar{x}\right)$. Then there exists $\bar{u} \in T(\bar{x}) \cap K$ such that $(\bar{x}-\bar{u})^{T}\left(x^{*}-\bar{x}\right)=0$, which implies that

$$
\left(\bar{x}-x^{*}\right)^{T}\left(x^{*}-\bar{x}\right)+\left(x^{*}-\bar{u}\right)^{T}\left(x^{*}-\bar{x}\right)=0 .
$$

Since $x^{*} \in S$, we have by Theorem 3.2 that $x^{*}$ is a solution of EP and so $x^{*} \in T\left(x^{*}\right)$. By monotonicity of $(-T)$, we have that the second term in (11) is non positive, which implies $-\left\|\bar{x}-x^{*}\right\|^{2}=\left(\bar{x}-x^{*}\right)^{T}\left(x^{*}-\bar{x}\right) \geq 0$, and so $\bar{x}=x^{*}$ is a solution of EP.

c) For each $x, y \in K$, we have

$$
\begin{aligned}
f(x, y) & =\sum_{i \in I}\left[f_{i}\left(x\left(y_{i}\right)\right)-f_{i}(x)\right] \\
& =\sum_{i \in I}\left[\left(f_{i i}\left(y_{i}\right)+\sum_{j \neq i} f_{i j}\left(x_{j}\right)\right)-\left(f_{i i}\left(x_{i}\right)+\sum_{j \neq i} f_{i j}\left(x_{j}\right)\right)\right] \\
& =\sum_{i \in I} f_{i i}\left(y_{i}\right)-\sum_{i \in I} f_{i i}\left(x_{i}\right) .
\end{aligned}
$$

By hypothesis, $0=f\left(\bar{x}, x^{*}\right)=\sum_{i \in I} f_{i i}\left(x_{i}^{*}\right)-\sum_{i \in I} f_{i i}\left(\bar{x}_{i}\right)$, and so $\sum_{i \in I} f_{i i}\left(x_{i}^{*}\right)=\sum_{i \in I} f_{i i}\left(\bar{x}_{i}\right)$. Hence, $f(\bar{x}, x)=\sum_{i \in I} f_{i i}\left(x_{i}\right)-\sum_{i \in I} f_{i i}\left(\bar{x}_{i}\right)=$ $\sum_{i \in I} f_{i i}\left(x_{i}\right)-\sum_{i \in I} f_{i i}\left(x_{i}^{*}\right)=f\left(x^{*}, x\right) \geq 0, \forall x \in K$.

d) We have that $0=f\left(\bar{x}, x^{*}\right)=\sup _{u \in T(\bar{x})} u^{T}\left(x^{*}-\bar{x}\right)$, then there exists $\bar{u} \in T(\bar{x})$ such that $\bar{u}^{T}\left(x^{*}-\bar{x}\right)=0$. Since $(T-I)$ is monotone, then $\left((\bar{u}-\bar{x})-\left(u^{*}-x^{*}\right)\right)^{T}\left(\bar{x}-x^{*}\right) \geq 0, \forall u^{*} \in T\left(x^{*}\right)$. Thus $\left(\bar{u}-u^{*}\right)^{T}\left(\bar{x}-x^{*}\right) \geq$ 
$\left\|\bar{x}-x^{*}\right\|^{2} \geq 0$, and so $0=\bar{u}^{T}\left(\bar{x}-x^{*}\right) \geq\left(u^{*}\right)^{T}\left(\bar{x}-x^{*}\right), \forall u^{*} \in T\left(x^{*}\right)$. We also have that $0 \leq f\left(x^{*}, \bar{x}\right)=\sup _{u^{*} \in T\left(x^{*}\right)}\left(u^{*}\right)^{T}\left(\bar{x}-x^{*}\right) \leq 0$, then there exists $\bar{u}^{*} \in T\left(x^{*}\right)$ such that $\left(\bar{u}^{*}\right)^{T}\left(\bar{x}-x^{*}\right)=0$. From (11), (12) and monotonicity of $(T-I)$, it follows that $-\left\|\bar{x}-x^{*}\right\|^{2}=\left(x^{*}-\bar{x}\right)^{T}\left(\bar{x}-x^{*}\right)=$ $\left((\bar{u}-\bar{x})-\left(\bar{u}^{*}-x^{*}\right)\right)^{T}\left(\bar{x}-x^{*}\right) \geq 0$, and so $\bar{x}=x^{*}$ is a solution of EP.

Now, we propose the following algorithm for identifying an $\epsilon$-solution of EP, which is a variant of the one proposed in [8].

\section{Algorithm}

Step 1 : (Initialization)

Given $\epsilon, \eta \in] 0,1\left[\right.$. Let $A^{0}=(I,-I) \in \mathbb{R}^{n \times 2 n}, b^{0}=\left(e^{T}, 0\right)^{T} \in \mathbb{R}^{2 n}$ and $P^{0}=\left\{x \in \mathbb{R}^{n}:\left(A^{0}\right)^{T} x \leq b^{0}\right\}$.

Set $k:=0$.

Step 2 : (Computation of an approximate analytic center)

Find $x^{k}$, an $\eta$-approximate analytic center of $P^{k}$.

Step 3 : (Stopping criterion)

Query the oracle to see if $x^{k} \in \operatorname{argmin}\left\{f\left(x^{k}, y\right): y \in[0,1]^{n}\right\}$. If the above inclusion is true, then stop;

Step $4:$ (Generation of the cutting plane)

Query the oracle to generate $T\left(x^{k}\right) \in \Gamma\left(x^{k}\right)$. Set:

$a^{k}:=T\left(x^{k}\right) /\left\|T\left(x^{k}\right)\right\|$,

$z^{k}:=b^{k}-\left(A^{k}\right)^{T} x^{k}$,

$\tau^{k}:=\sqrt{\left(a^{k}\right)^{T}\left(A^{k}\left(Z^{k}\right)^{-2}\left(A^{k}\right)^{T}\right)^{-1} a^{k}}$

$\rho^{k}:=\sqrt{\left(a^{k}\right)^{T} A^{k}\left(Z^{k}\right)^{-2}\left(A^{k}\right)^{T} a^{k}}$

$\beta^{k}:=\min \left\{\frac{\eta}{(1-\eta)^{2}}-\frac{\eta^{2}}{2(1-\eta)}, \frac{1-\eta}{2 \rho^{k} \tau^{k}}\right\}$.

Update:

$A^{k+1}=\left(A^{k}, a^{k}\right), \quad b^{k+1}=\left(\left(b^{k}\right)^{T},\left(a^{k}\right)^{T} x^{k}+\beta^{k} \tau^{k}\right)^{T}$ and

$P^{k+1}=\left\{x \in \mathbb{R}^{n}:\left(A^{k+1}\right)^{T} x \leq b^{k+1}\right\}$.

Set $k:=k+1$ and return to step 2 .

\section{End}

In step 4 of the above algorithm, the updating of $x^{k}$ after a cut is done, as described at the end of Section 2, is assumed as part of the procedure of finding a new analytic center. The following lemmas show that the algorithm above is well defined. We skip the proofs of those lemmas that follow straightforward.

Lemma 4.2. Let $x^{k}$ be generated by the algorithm. If $T\left(x^{k}\right)=0$, then

$$
x^{k} \in \operatorname{argmin}\left\{f\left(x^{k}, y\right): y \in[0,1]^{n}\right\} .
$$

Moreover, $x^{k}$ is a solution of EP.

Lemma 4.3. Let $x^{k}$ be generated by the algorithm. If $T\left(x^{k}\right) \neq 0$, then $\tau^{k}>0$, $\rho^{k}>0$ and $\beta^{k}>0$. 
Lemma 4.4. Let $x^{k}$ be generated by the algorithm. If $x^{k} \notin \operatorname{argmin}\left\{f\left(x^{k}, y\right): y \in\right.$ $\left.[0,1]^{n}\right\}$, then:
a) $T\left(x^{k}\right) \neq 0$;
b) $S \subset P^{k}$. Moreover, $T\left(x^{k}\right)^{T}\left(x-x^{k}\right)<0 \quad \forall x \in S$.

Proof.

a) It follows directly from the contrapositive result of Lemma 4.2 .

b) We know that

$$
A^{k}=\left(A^{0}, a^{0}, \ldots, a^{k-1}\right) \text { and } b^{k}=\left(\begin{array}{c}
b^{0} \\
\left(a^{0}\right)^{T} x^{0}+\beta^{0} \tau^{0} \\
\vdots \\
\left(a^{k-1}\right)^{T} x^{k-1}+\beta^{k-1} \tau^{k-1}
\end{array}\right) .
$$

So, we only have to prove that for any $x \in S,\left(A^{k}\right)^{T} x<b^{k}$. Since $x \in S \subset K \subset$ ]0, $1^{n}$, then $\left(A^{0}\right)^{T} x<b^{0}$. By Theorem 3.3, we have

$$
\left(a^{i}\right)^{T}\left(x-x^{i}\right)=\left(\frac{T\left(x^{i}\right)}{\left\|T\left(x^{i}\right)\right\|}\right)^{T}\left(x-x^{i}\right) \leq 0 \forall i \in\{0, \ldots, k-1\} .
$$

So, $\left(a^{i}\right)^{T} x \leq\left(a^{i}\right)^{T} x^{i}, \forall i \in\{0, \ldots, k-1\}$. Since $a^{i}=T\left(x^{i}\right) /\left\|T\left(x^{i}\right)\right\| \neq 0$, then by Lemma $4.3 \tau^{i} \beta^{i}>0, \forall i \in\{0, \ldots, k-1\}$. It implies $\left(a^{i}\right)^{T} x \leq\left(a^{i}\right)^{T} x^{i}<$ $\left(a^{i}\right)^{T} x^{i}+\beta^{i} \tau^{i}, \forall i \in\{0, \ldots, k-1\}$. And so $\left(A^{k}\right)^{T} x<b^{k}$.

Finally, suppose there exists $x^{*} \in S$ such that $T\left(x^{k}\right)^{T}\left(x^{*}-x^{k}\right)=0$, then $0=$ $T\left(x^{k}\right)^{T}\left(x^{*}-x^{k}\right) \leq f\left(x^{k}, x^{*}\right) \leq 0$. It follows by assumption A4 that $x^{k}$ is a solution of EP, which is a contradiction, because $x^{k} \notin \operatorname{argmin}\left\{f\left(x^{k}, y\right): y \in[0,1]^{n}\right\}$.

Lemma 4.5. Let $x^{k}$ be generated by the algorithm. If $T\left(x^{k}\right) \neq 0$, then the hyperplane

intersects the Dikin ellipsoid

$$
H^{k}=\left\{x \in \mathbb{R}^{n}:\left(a^{k}\right)^{T}\left(x-x^{k}\right)=\beta^{k} \tau^{k}\right\}
$$

$$
E^{k}=\left\{x \in \mathbb{R}^{n}:\left\|\left(Z^{k}\right)^{-1}\left(A^{k}\right)^{T}\left(x-x^{k}\right)\right\| \leq 1-\eta\right\} .
$$

Proof. It follows from the fact that $\left.\beta^{k} \leq \frac{1-\eta}{2 \rho^{k} \tau^{k}} \in\right] 0, \frac{1-\eta}{\rho^{k} \tau^{k}}[$ and applying Lemma 2.1.

Using the same argument as in Goffin et al. [8] we find an upper bound for $\sum_{i=0}^{k}\left(\tau^{i}\right)^{2}$. This is achieved by a construction from Nesterov [14], which bounds $A^{k}\left(Z^{k}\right)^{-2}\left(A^{k}\right)^{T}$ from below by a certain matrix $B^{k}$ simple enough to handle. We reproduce this result in the next lemma.

Lemma 4.6. Let $x^{k}$ be generated by the algorithm. If $T\left(x^{k}\right) \neq 0$, then

$$
\sum_{i=0}^{k}\left(\tau^{i}\right)^{2} \leq 2 n^{2} \ln \left(1+\frac{k+1}{8 n^{2}}\right) .
$$


Theorem 4.7. Given $\epsilon>0$ and $L>\sup _{x \in K}\{\|v\|: v \in \Gamma(x)\}$, the algorithm finds an $\epsilon$-solution for EP (1) as soon as $k$ satisfies one of the following conditions:

(1) $x^{k} \in \operatorname{argmin}\left\{f\left(x^{k}, y\right): y \in[0,1]^{n}\right\}$,

(2) $\frac{\epsilon^{2}}{L^{2} n} \geq \frac{\frac{1}{2}+2 n \ln \left(1+\frac{k+1}{8 n^{2}}\right)}{2 n+k+1} \exp \left(-2 \alpha \frac{k+1}{2 n+k+1}\right)$,

where $\alpha=-\ln (4)-(8 \eta-3) /\left(2(1-\eta)^{2}\right)$.

Proof.

(1) If there exists $k \in \mathbb{N}$ such that $x^{k} \in \operatorname{argmin}\left\{f\left(x^{k}, y\right): y \in[0,1]^{n}\right\}$, then by Lemma $4.2 x^{k} \in S$.

(2) If $x^{k} \notin \operatorname{argmin}\left\{f\left(x^{k}, y\right): y \in[0,1]^{n}\right\} \forall k \in \mathbb{N}$, then by Lemma $4.4 S \subset \stackrel{o}{k}$ $\forall k \in \mathbb{N}$, and $T\left(x^{k}\right)^{T}\left(x-x^{k}\right)<0, \forall x \in S$ and $\forall k \in \mathbb{N}$. Note that by construction $\stackrel{o}{P^{k+1}} \subset \stackrel{o}{P^{k}}, \forall k \in \mathbb{N}$.

Now, consider $k \in \mathbb{N}$ such that

$$
\begin{aligned}
\phi\left(x^{k+1}\right) & =\sum_{j=1}^{2 n+k+1} \ln \left[\left(b^{k+1}-\left(A^{k+1}\right)^{T} x^{k+1}\right)_{j}\right] \\
& \geq \sum_{j=1}^{2 n+k+1} \ln \left[\left(b^{k+1}-\left(A^{k+1}\right)^{T} x\right)_{j}\right] \\
& \geq \sum_{j=1}^{2 n+k+1} \ln \left[\left(b_{0}^{k+1}-\left(A^{k+1}\right)^{T} x\right)_{j}\right] \\
& \geq \sum_{j=1}^{2 n+k+1} \ln (\epsilon / L) \quad \forall x \in S .
\end{aligned}
$$

According to definition of $S(\delta=\epsilon / L)(10)$, the last inequality comes from the fact that

where

$$
S(\epsilon / L)=S+B(0, \epsilon / L) \subset \stackrel{o}{P_{0}^{k}}
$$

with

$$
P_{0}^{k}=\left\{x \in \mathbb{R}^{n}:\left(A^{k}\right)^{T} x \leq b_{0}^{k}\right\}
$$

$$
\left(b_{0}^{k+1}\right)_{2 n+j}=\left(a^{j-1}\right)^{T} x^{j-1} \quad \forall j=1, \ldots, k+1 .
$$

On the other hand, from (7) and from (9), we have

$$
\phi\left(x^{k+1}\right) \leq \phi\left(\bar{x}^{a}\right) \leq \phi\left(x^{a}\right)+\ln \left(4 \tau^{k}\right)-\left(1.5-\beta^{k}\right) .
$$

We also have from (7) that

$$
\phi\left(x^{a}\right) \leq \phi\left(x^{k}\right)+\frac{\eta^{2}}{2(1-\eta)},
$$


thus

Since

$$
\phi\left(x^{k+1}\right) \leq \phi\left(x^{k}\right)+\ln \left(\tau^{k}\right)+\ln (4)-1.5+\beta^{k}+\frac{\eta^{2}}{2(1-\eta)} .
$$

we have

$$
\beta^{k}=\min \left\{\frac{\eta}{(1-\eta)^{2}}-\frac{\eta^{2}}{2(1-\eta)}, \frac{1-\eta}{2 \rho^{k} \tau^{k}}\right\}
$$

$$
\phi\left(x^{k+1}\right)-\phi\left(x^{k}\right) \leq \ln \left(\tau^{k}\right)+\ln (4)-1.5+\frac{\eta}{(1-\eta)^{2}} .
$$

Thus

$$
\phi\left(x^{k+1}\right)-\phi\left(x^{k}\right) \leq \frac{1}{2} \ln \left(\tau^{k}\right)^{2}+\ln (4)+\frac{8 \eta-3}{2(1-\eta)^{2}} .
$$

Let $\alpha=-\ln (4)-(8 \eta-3) /\left(2(1-\eta)^{2}\right)$, then

$$
\phi\left(x^{k+1}\right)-\phi\left(x^{k}\right) \leq \frac{1}{2} \ln \left(\tau^{k}\right)^{2}-\alpha
$$

So

or

$$
\sum_{j=0}^{k}\left(\phi\left(x^{j+1}\right)-\phi\left(x^{j}\right)\right) \leq \frac{1}{2} \sum_{j=0}^{k} \ln \left(\tau^{j}\right)^{2}-(k+1) \alpha
$$

$$
\phi\left(x^{k+1}\right)-\phi\left(x^{0}\right) \leq \frac{1}{2} \sum_{j=0}^{k} \ln \left(\tau^{j}\right)^{2}-(k+1) \alpha .
$$

Hence

$$
\phi\left(x^{k+1}\right)+(k+1) \alpha \leq \phi\left(x^{0}\right)+\frac{1}{2} \sum_{j=0}^{k} \ln \left(\tau^{j}\right)^{2}=2 n \ln \left(\frac{1}{2}\right)+\frac{1}{2} \sum_{j=0}^{k} \ln \left(\tau^{j}\right)^{2} .
$$

From (13), we have

$$
(2 n+k+1) \ln (\epsilon / L)+(k+1) \alpha \leq \frac{1}{2}\left[2 n \ln \left(\frac{1}{4}\right)+\sum_{j=0}^{k} \ln \left(\tau^{j}\right)^{2}\right] .
$$

Then

$$
\ln (\epsilon / L)+\frac{k+1}{2 n+k+1} \alpha \leq \frac{1}{2(2 n+k+1)}\left[2 n \ln \left(\frac{1}{4}\right)+\sum_{j=0}^{k} \ln \left(\tau^{j}\right)^{2}\right] .
$$

From concavity of $\ln$, we have

$$
\ln (\epsilon / L)+\frac{k+1}{2 n+k+1} \alpha \leq \frac{1}{2} \ln \left[\frac{2 n \frac{1}{4}}{2 n+k+1}+\frac{\sum_{j}^{k}\left(\tau^{j}\right)^{2}}{2 n+k+1}\right]
$$


and from Lemma 4.6, we have

$$
\ln (\epsilon / L)+\frac{k+1}{2 n+k+1} \alpha \leq \frac{1}{2} \ln \left[\frac{\frac{n}{2}+2 n^{2} \ln \left(1+\frac{k+1}{8 n^{2}}\right)}{2 n+k+1}\right] .
$$

Thus,

$$
\frac{\epsilon^{2}}{L^{2} n} \leq \frac{\frac{1}{2}+2 n \ln \left(1+\frac{k+1}{8 n^{2}}\right)}{2 n+k+1} \exp \left(-2 \alpha \frac{k+1}{2 n+k+1}\right) .
$$

As a consequence of inequality (14), we have that for each $\epsilon>0$ and $L>\sup _{x \in K}\{\|v\|: v \in \Gamma(x)\}$, and according to definition $S(\delta)$, there exists $k \in \mathbb{N}$ such that $S(\epsilon / L) \subset \stackrel{o}{P_{0}^{k}}$, but $S(\epsilon / L) \not \subset P_{0}^{k+1}$. And thus, for

$$
H_{0}^{k+1}:=\left\{x \in \mathbb{R}^{n}:\left(a^{k+1}\right)^{T}\left(x-x^{k+1}\right)\right\}
$$

we have that

$$
H_{0}^{k+1} \cap S(\epsilon / L) \neq \emptyset .
$$

So, as $L \rightarrow+\infty$ or $\epsilon \rightarrow 0$, we have

$$
\lim _{k \rightarrow+\infty} d\left(H_{0}^{k}, S\right)=\lim _{k \rightarrow+\infty} \inf _{x \in H_{0}^{k}, y \in S}\|x-y\|=0 .
$$

Hence, there exists $x^{*} \in S$ such that $\lim _{k \rightarrow+\infty}\left(a^{k}\right)^{T}\left(x^{*}-x^{k}\right)=0$. Since $f$ is bounded above, then by item c of Lemma 3.4 the sequence $\left\{T\left(x^{k}\right)=\left\|T\left(x^{k}\right)\right\| a^{k}\right\}_{k \in \mathbb{N}}$ is bounded. So, $\lim _{k \rightarrow+\infty} T\left(x^{k}\right)^{T}\left(x^{*}-x^{k}\right)=0$. Let $\bar{x}$ be any cluster point of $\left\{x^{k}\right\}$, then there exists $J \subset \mathbb{N}$ such that $x^{k} \rightarrow \bar{x}$, and using Lemma $3.1 T\left(x^{k}\right) \rightarrow T(\bar{x})$, as $k \rightarrow+\infty$ and $k \in J$. And so, $0=\lim _{k \rightarrow+\infty, k \in J} T\left(x^{k}\right)^{T}\left(x^{*}-x^{k}\right)=T(\bar{x})^{T}\left(x^{*}-\bar{x}\right) \leq$ $f\left(\bar{x}, x^{*}\right) \leq 0$. Hence, using assumption A4, we have that $\bar{x}$ is a solution of EP. The algorithm stops at iteration $k$ when inequality (14) does not hold and $d\left(x^{k+1}, S\right) \leq \epsilon / L$, verifying that $T(y)^{T}\left(x^{k+1},-y\right)<\epsilon \forall y \in K$, which validates Theorem 3.5.

\section{Final COmments}

Here we present a new approach to solve equilibrium problems approximately, which include as particular problems the variational inequalities problem, the Nash equilibria problem in non-cooperative games, the convex minimization problem, and the fixed point problem. Without using projection techniques and considering different hypotheses compared to the existing methodology in literature, we proposed an algorithm that combines the cutting plane method with the technique of interior point methods which allows for feasible computer implementation with polynomial time complexity. In future work, we want to adjust the algorithm proposed here in order to solve multicriteria optimization problems, where the objective functions as well as the constrains are linear functions. 


\section{REFERENCES}

[1] A.S. Antipin and F.P. Vasil'ev, A stabilization method for equilibrium programming problems with an inexactly specified set. Comp. Math. Math. Phys. 39 (1999) 1707-1714.

[2] A.S. Antipin, From Optima to Equilibria, in Proceedings of ISA RAS, Dynamics of NonHomogeneous Systems. Editorial URSS-Moscow 3 (2000) 35-64.

[3] Bianchi-Pini, A note on equilibrium problems with properly quasimonotone bifunctions. $J$. Global Optim. 20 (2001) 67-76.

[4] E. Blum and W. Oettli, From optimization and variational inequalities to equilibrium problems. The Mathematics Student 63 (1994) 123-145.

[5] L.M. Bregman, The relaxation method for finding a common point of convex sets and its applications to solution of problems in convex programming. USSR Comp. Math. Math. Phys. 7 (1967) 200-217.

[6] H. Brezis, L. Niremberg and G. Stampachia, A remark on Ky Fan's minimax principle. Boll. Un. Mat. Ital. 6 (1972) 293-300.

[7] K. Fan, A minimax inequality and applications, in Inequality III, edited by O. Shisha. Academic Press, NY (1972) 103-113.

[8] J.-L. Goffin, Z.-Q. Luo and Y. Ye, Complexity analysis of an interior cutting plane method for convex feasibility pProblems. SIAM J. Optim. 6 (1996) 638-652.

[9] J.-L. Goffin, J. Gonzio, R. Sarkissian and J.-P. Vial, Solving nonlinear multicommodity flow problems by analytic center cutting plane method. Interior point methods in theory and practice. Math. Program. Ser. B 76 (1997) 131-154.

[10] C.C. Gonzaga, Path following methods for linear programming. SIAM Rev. 34 (1992) 167224.

[11] G.M. Korpelevich, Extragradient method for finding saddle points and other problems. Matecon 12 (1976) 747-756.

[12] A.N. Iusem and W. Sosa, New existence results for equilibrium problems. Nonlinear Anal.Theor. 52 (2003) 621-635.

[13] A.N. Iusem and W. Sosa, Iterative algorithms for equilibrium problems. Optimization 52 (2003) 301-316.

[14] Y. Nesterov, Complexity estimates of some cutting plane methods based on the analytic barrier. Nondifferentiable and large-scale optimization. Math. Program. Ser. B 69 (1995) $149-176$.

[15] H. Nikaido and K. Isoda, Note on noncooperative convex games. Pacific J. Math. 5 (1955) 807-815.

[16] F.M.P. Raupp and C.C. Gonzaga, A center cutting plane algorithm for a likelihood estimate problem. Comput. Optim. Appl. 21 (2001) 277-300.

[17] G. Sonnevend, New algorithms in convex programming based on a notation of center and on rational extrapolations. International Series of Numerical Mathematics, Birkhauser Verlag, Basel, Switzerland 84 (1988) 311-327.

[18] P.M. Vaidya, A Locally Well-Behaved Potential Function and a Simple Newton-Type Method for Finding the Center of a Polytope. Progress in Mathematical Programming: Interior Point and Related Methods, edited by N. Megiddo. Springer, New York (1989) 79-90.

[19] Y. Ye, A potential reduction algorithm allowing column generation. SIAM J. Optim. 2 (1992) 7-20.

[20] Y. Ye, Complexity analysis of the analytic center cutting plane method that uses multiple cuts. Math. Program. 78 (1997) 85-104.

[21] Y. Ye, Interior Point Algorithms: Theory and Analysis. Wiley-Interscience Series in Discrete Mathematics and Optimization, John Wiley and Sons, New York (1997). 